WALL CLASSIFIER FOR GONDOLA-TYPED ROBOT USING KALMAN FILTER

*D. Y. Kim, J. Yoon, and C.-W. Park Korea Electronics Technology Institute 193, Yakdae-dong, Wonmi-gu

Bucheon-si, Gyeonggi-do, Republic of Korea

(*Corresponding author: sword32@keti.re.kr) 


\title{
WALL CLASSIFIER FOR GONDOLA-TYPED ROBOT USING KALMAN FILTER
}

\begin{abstract}
This paper shows an approach that a gondola-typed robot recognizes walls on building facades. It is applicable for autonomous or teleoperated building façade painting robots. The robot may react based on the wall classification data. This approach is based on 2D LRF(two-dimensional Laser Range Finder). The robot acquires relative distances to walls, and a Kalman filter decides correspondence of walls. An ARS(Attribute Reference System) sensor helps convert LRF coordinate to global coordinate. The experimental results demonstrate the conversion of LRF data.
\end{abstract}

\section{KEYWORDS}

Robotics, Gondola, Façade, Maintenance, Wall, Classifier

\section{INTRODUCTION}

As social safety becomes important, cost for saving human lives from dangerous workspace is more acceptable. Robots in construction are good examples for that. As one of this dangerous workplace, building façade has caused a lot of accidents. We have researched automated gondola-typed robot systems to solve this problem (Sun et al., 2011; Kim et al., 2012).

We suggest recognition methodology to help the gondola robot more intelligent, and more people work at safe working place. It is a wall classification methodology. For control strategy, the state of the wall is an important factor. For automatically controlled painting on the wall, window detection is a critical issue (Kim et al., 2012). With suggest methodology, the walls can be detected and classified with laser scanner.

In this paper, we will introduce our robot platform and scenario. Then, the role of LRF and Kalman filter will be described. With experimental results, LRFs calibration and wall classification will be discussed.

\section{METHODS}

\section{Gondola-typed Robot Platform and Scenario}

Fig. 1 is our gondola-typed robot platform. There is not any space for human boarding because it is automated. On the other hand, many sensors and actuators enable automation mode and teleoperation mode. Two endless winders make the gondola navigate on two wire ropes. Two suction fans keep contact between the gondola body and the wall. As a sensor system, the gondola uses an ARS sensor to maintain balance. Two encoders measure the amount of navigation on the wire ropes. 


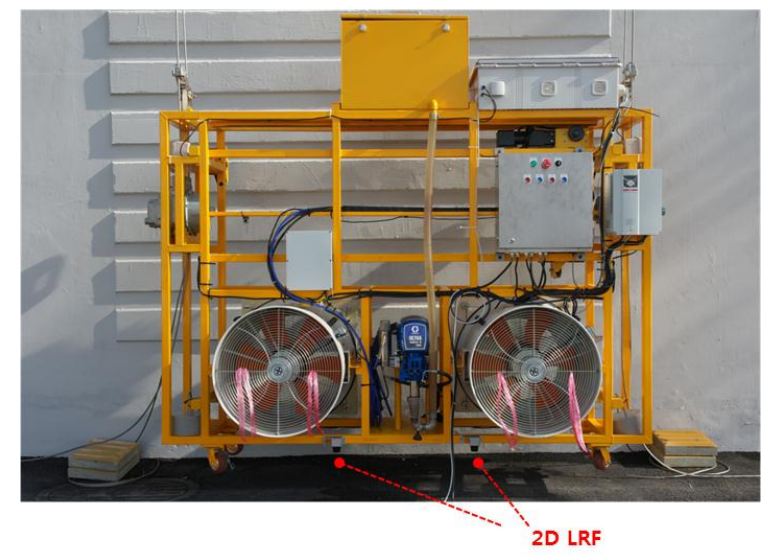

Figure 1 - Gondola- typed robot platform with two 2D laser range finder

It has two 2D LRFs(two-dimensional Laser Range Finders). Their location was selected in accordance with the scenario of automated gondola-typed robot. As first step of the scenario, the gondola goes top of the wall. Then, the gondola begins to go down while painting is executed. Therefore, the LRFs are installed at the bottom frame as shown in Fig. 1. They sense the state of the wall $80 \mathrm{~cm}$ earlier than painting tools.

The reason that two LRF are applied to our system is blocking of the FoV(Field of View) of LRF by wheels. However, the wheels cannot be eliminated because they support the gondola frame when the gondola is on the ground. The two LRFs complement each other to overcome this limitation. In order to build valid data, they should be synthesized. We designed two LRF calibrations methodology, and its detail will be shown in experimental discuss.

\section{Laser Range Finder Line Extraction}

Because our system is a reinforced concrete building maintenance robot, target wall is plat. Therefore, line detection is appropriate to our system. We use Split-and-Merge algorithm to interpret point data of LRFs to line data (Nguyen et al., 2005). This algorithm extracts points neighboring and aligning on straight line, and makes the lines described with parameters.

The gondola-typed robot can rotate on rolling and pitching axis. Unlike 3D sensor such as Kinect, it is difficult to recognise such rotations only with 2D LRF. We applied an ARS (Attitude Reference System) sensor to get roll and pitch values. The values are converted to rotation matrix for preprocess of 2D LRF data.

Even though roll and pitch are sensed by our sensor system, yaw is not measured. The main reason is that suction fans on gondola system make the contact between the gondola and the wall be kept. For plat surface of the wall, the yaw does not fluctuate.

\section{Kalman Filter}

We assumed that the robot contact on the wall firmly at the beginning of scenario. Then, the location of the robot is initialized as zero and registered on the Kalman filter. Relative distances from the robot to walls are also registered on the Kalman filter like landmarks of SLAM (Simultaneous Localization and Mapping) (Thrun et al., 2005). As the gondola moves down, the location of the robot and relative distances between the robot and walls are updated (Davison et al., 2007). The state vector of Kalman filter is

$$
\mathbf{X}_{t}=\left[\begin{array}{lllll}
\mathbf{x}_{t} & \mathbf{w}_{t}^{1} & \mathbf{w}_{t}^{2} & \cdots & \mathbf{w}_{t}^{N}
\end{array}\right]
$$


$\mathbf{x}_{t}$ is the location of the robot on the vertical direction from initial wall plane. $\mathbf{w}_{t}^{n}$ is the significance of $n$th walls. It is vertical distance wall plane to the initial wall plane. Basically, it is simpler than other six DoF (Degree of Freedom) application. The robot has only one DoF, and each wall also has only one DoF.

\section{RESULTS}

\section{Line Extraction and Calibration}

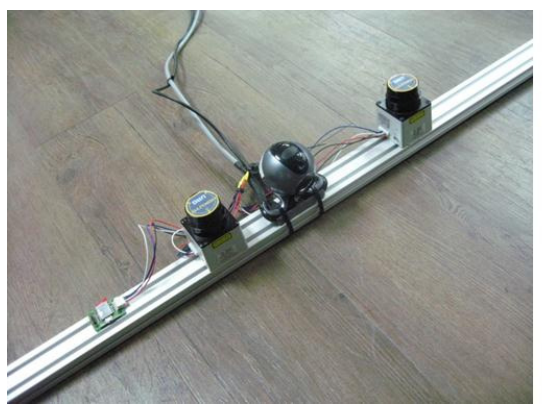

(a)

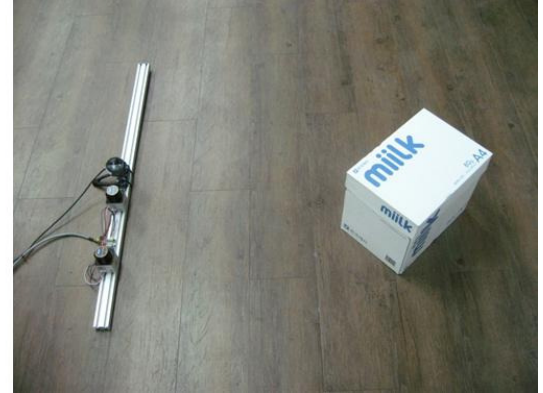

(b)

Figure 2 - (a) Two 2D LRF, an ARS sensor, and a monitoring webcam

(b) A rectangular box as calibration reference

We used laser scanner, Hokuyo URG-04lx. It uses semiconductor laser diode (wave length $\lambda=$ $785 \mathrm{~nm}$ ). Fig. 2(a) shows the experimental setup. There are two 2D LRF, one ARS, and one monitoring webcam. Base on the aluminum profile support, movement of theses sensors are coincide. Fig. 2(b) is calibration target of two 2D LRFs. Among extracted lines from 2D LRFs, only suitable lines for this box are used for the calibration phase. With above setup, output is shown in fig. 3. The smallest green circle on LRF output is one meter radius circle. There is a shape of a rectangular box.

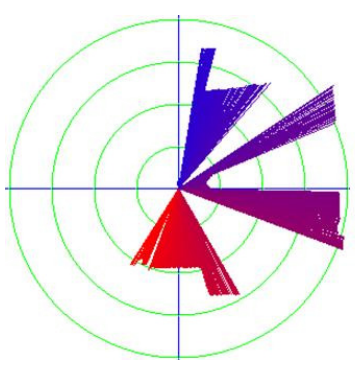

(a)

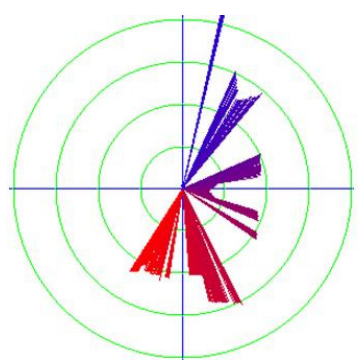

(b)

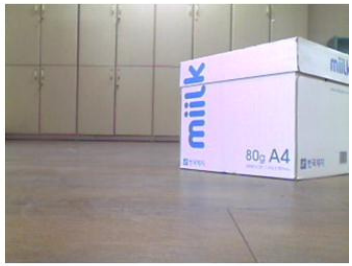

(c)

Figure 3 - (a) Output of first 2D LRF (b) Second LRF (c) Webcam monitoring as reference

With split-and-merge and LRF range limitation, only two line segments for each LRF sensor can be extracted because we knows the size of the box. Lines of different length are discussed. Even though the locations on each LRF outputs are different, it is the same object. The difference is the locational difference of two LRF.

The gap is calibrated with following simple steps. First, two lines on each output are converted to vectors. Second, the cross points of two vectors are assumed as the same point. Third, all vectors are 
normalized. Finally, using the cross point as a center, rotation matrix is selected through least error of end points of normalized vectors.

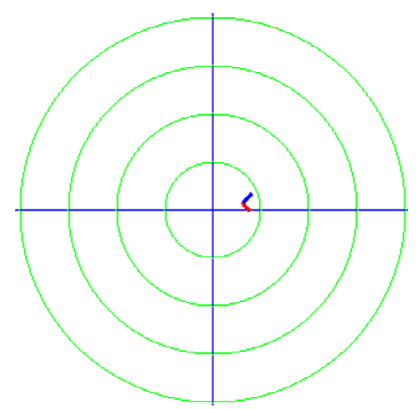

(a)

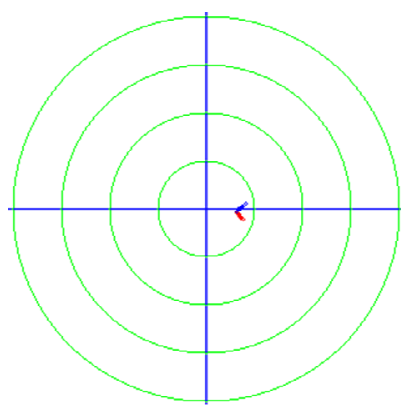

(b)

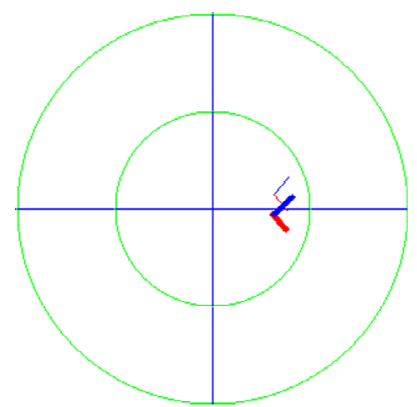

(c)

Figure 4 - (a) Split-and-merge output of first LRF (b) Second LRF output (c) Overlay of two outputs

\section{Line Extraction with Rolling and Pitching}
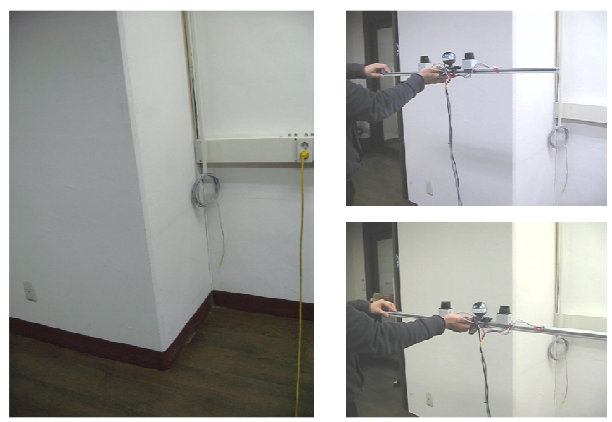

Figure 5 - Wall classification test

Fig. 5 shows the experiment for walls. As shown in Fig. 5, data gathering was conducted while the jig is hand-held. The roll and pitch fluctuated. Therefore, they should be reflected on LRF output every time. Before applying LRF output to Kalman filter, the LRF data was converted to pure $x-y$ plane data with inverse rotation matrix. Fig. 6 shows the difference of raw LRF output and pure $x-y$ plane LRF output. Because the proposed wall classification uses only the horizontal distance between gondola and walls, roll and pitch can be critical error source.

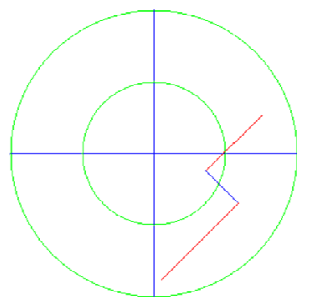

(a)

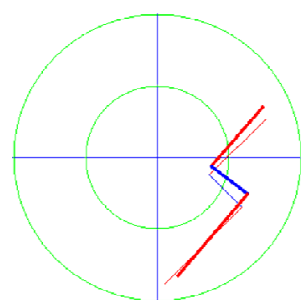

(b)

Figure 6 - (a) Raw LRF output (b) Pure x-y plane LRF output (overlaid bold lines)

\section{DISCUSSION}


We have researched the window detection problem (Kim et al., 2012). If a malfunction occurs and the robot paints on windows, cost may be great. However, there are too many cases for windows. For example, some glass is clear and other glass is covered with tinting. Safety grille may cover a window. Color and material cannot be limited. We tried to use a characteristic of glass that ultrasonic is reflected and infrared pass through. However, dust and tinting on glass disturb this characteristic.

In this paper, we adopt another approach. It is wall detection. Contrast to window, the wall usually has consistent shape. It is flat and wide. That is the reason that we use LRF sensor to extract wall lines and wall classifier.

\section{CONCLUSIONS}

An algorithm for autonomous or teleoperated gondola-typed robot system is proposed. It is a wall classifier to help the robot recognize the wall and select its reaction on it. The most anxious situation is that the robot paints on window glass. The proposed algorithm was designed to prevent this situation. LRF sensors are used to detect walls using the characteristic that wall is straight and wide. In order to make LRF output accurate, an ARS sensor is applied. It removes the effect of roll and pitch. The extracted distances between walls and the robot are input to Kalman filter using concept of SLAM.

As a future work, we want to use a visual camera as data source, and fuse with LRF data. If we synthesis them like Kinect, human robot interface for robot operators will be more abundant.

\section{ACKNOWLEDGEMENTS}

The work presented in this paper was funded by BMRC (Building-Facade Maintenance Robot Research Center), supported by Korea Agency for Infrastructure Technology Advancement (KAIA) under the Ministry of Land, Infrastructure and Transport (MOLIT).

\section{REFERENCES}

Davision, A.J., Reid, I.D., Molton, N.D., \& Stasse, O. (2007). MonoSLAM: Real-Time Single Camera SLAM. IEEE Trans. Pattern Analysis and Machine Intelligence, Vol. 29, no. 6, pp. 1052-1067.

Kim, D. Y., Sun, H., Yoon, J., \& Park, C.-W. (2012). Height estimation of gondola-typed facade robot. In Proceedings of 29th International Symposium on Automation and Robotics in Construction (ISARC 2012).

Kim, D. Y., Yoon, J., Sun, H., \& Park, C.-W. (2012). Window detection for gondola robot using a visual camera. In Proceeding of IEEE International Conference on Automation Science and Engineering (CASE 2012).

Nguyen, V.,Martinelli, A., Tomatis, N., \& Siegwart, R. (2005). A comparison of line extraction algorithms using $2 \mathrm{~d}$ laser rangefinder for indoor mobile robotics. In Proceedings of IROS '05.

Sun, H., Kim, D. Y., Kwon, J. H., Kim, B.-S., \& Park, C.-W. (2011). The position and orientation measurement of gondola using a visual camera. In Proceeding of 28th International Symposium on Automation and Robotics in Construction (ISARC 2011).

Thrun, S., Burgard, W., \& Fox., D. (2005). Probabilistic Robotics. MIT Press. 